\title{
Censo local sobre consumo de drogas en población escolar
}

\author{
Carolina Espinosa-Luna(D), María del Rocío Hernández-Pozo(D2², Marco Torres-Inguanzo(D)3, Boris González-Ceja(D4 \\ I Programa de Gobierno y Políticas Públicas, Centro Regional de Investigaciones Multidisciplinarias, Universidad Nacional Autónoma de México. \\ ${ }^{2}$ Laboratorio de Felicidad y Bienestar Subjetivo, Centro Regional de Investigaciones Multidisciplinarias y Grupo de Investigación Aprendizaje Humano, \\ Facultad de Estudios Superiores Iztacala, Universidad Nacional Autónoma de México. \\ 3 Unidad Académica en Desarrollo y Gestión Pública de la Universidad Autónoma de Zacatecas. \\ ${ }^{4}$ Asociación Mexicana de Psicología y Desarrollo Comunitario A.C.
}

\section{RESUMEN}

Introducción: bajo un marco de prevención social de la violencia se presentan resultados preliminares de un censo sobre consumo de drogas en población escolar en una localidad del municipio de Guadalupe, Zacatecas, México. Objetivo: conocer la prevalencia del consumo de drogas en la población escolar de la localidad. Método: se levantó un censo sobre consumo de drogas con el cuestionario elaborado por el INPRFM para la ENCODE 2014 en su versión para población escolar de secundaria y bachillerato. El cuestionario fue respondido por $96.6 \%$ de la población estudiada ( $N=1139$ ). Resultados: $33.3 \%$ de los estudiantes afirmó que les han ofrecido drogas regaladas y $7.9 \%$ afirmó que les han ofrecido drogas vendidas. Las prevalencias de consumo alguna vez en la vida fueron de $49.4 \%$ para alcohol, $37.6 \%$ para tabaco y $24.3 \%$ para drogas como anfetaminas, tranquilizantes, mariguana, cocaína, crack o piedra, inhalables, metanfetaminas o heroína. Las prevalencias de consumo en los últimos 12 meses fueron de 56.9\% para alcohol, $20.1 \%$ para tabaco y $13.2 \%$ para otras drogas. Las prevalencias de consumo en el último mes fueron de 35.8\% para alcohol, $9.1 \%$ para tabaco y $9 \%$ para otras drogas. Discusión: los resultados preliminares del censo sugieren la necesidad de identificar factores asociados al consumo de drogas en las escuelas de la localidad. Un análisis exploratorio de regresión logística bivariado podría ser pertinente para estudiar la relación entre las características personales o sociodemográficas y el consumo de drogas. Se proponen dos rutas de acción, una de tipo psicosocial para delimitar necesidades y diseñar intervenciones breves adaptadas a la población, y otra de tipo sociopolítico para intervenir en el contexto social de conflicto e ilegalidad que genera la accesibilidad a las drogas.

Palabras clave: conducta adictiva, consumo de drogas en menores, prevención.

\begin{abstract}
Introduction: Under the framework of social prevention of violence, the results of a survey on drug use in school population in a locality in Guadalupe, Zacatecas, Mexi$\mathrm{co}$, are presented. Objective: Determine the prevalence of drug consumption in the local student population. Method: The questionnaire developed by INPRFM for ENCODE 2014 in its version for student age populations was applied. The questionnaire was answered by $96.6 \%$ of the population studied ( $N=1139)$. Results: $33.3 \%$ of the students stated that they had been offered drugs as gifts and $7.9 \%$ stated that they had been offered to buy drugs. Lifetime prevalence were $49.4 \%$ for alcohol, $37.6 \%$ for tobacco and $24.3 \%$ for some drugs such as amphetamines, tranquilizers, marijuana, cocaine, crack or rock, inhalants, methamphetamines or heroin. Prevalence of use in the last twelve months were $56.9 \%$ for alcohol, $20.1 \%$ for tobacco and $13.2 \%$ for other drugs. Prevalence of use in the last month were $35.8 \%$ for alcohol, $9.1 \%$ for tobacco and $9 \%$ for other drugs. Discussion: the relevance of a bivariate logistic regression analysis is proposed to study the connection between personal or sociodemographic characteristics with drug use. Two routes of action are proposed: a) Psychosocial, brief interventions adapted to the population and b) Socio-political, influence on the social context of conflict and illegality.
\end{abstract}

Keywords: Addictive behavior, use of drugs by minors, prevention.

\footnotetext{
Autor de correspondencia:

Carolina Espinosa-Luna. CRIM-UNAM. Av. Universidad s/n, Circuito 2, 62210, Col. Chamilpa, Ciudad Universitaria de la UAEM. Cuernavaca, Morelos. Teléfono: 55562 27829. Email: espinosa@crim.unam.mx

Recibido: 28 de julio de 2020

Aceptado: 5 de mayo de 2021

doi: 10.28931/riiad.2021.2.05
} 


\section{INTRODUCCIÓN}

En América Latina, la discusión académica sobre el tema de inseguridad ha transitado de un enfoque de seguridad pública, centrado en el estado, a uno de seguridad ciudadana, que parte de supuestos de corresponsabilidad entre gobierno y sociedad (Carrión, 2005; Dammert, 2007; Rico \& Chinchilla, 2002). En términos de políticas públicas sobre prevención de la violencia, esta transición ha implicado pasar de un enfoque punitivo a uno de prevención social (Welsh \& Farrington, 2012) que busca atender los factores de riesgo asociados al delito y la violencia en contextos locales (Oficina de Naciones Unidas contra la Droga y el Delito, 2011).

Bajo esas premisas, se encuentra en curso un proyecto de investigación que tiene como propósito diagnosticar, diseñar, ejecutar y evaluar una estrategia de intervención psicosocial para disminuir tres factores de riesgo asociados al delito y la violencia: el consumo de drogas (Fergusson \& Horwood, 2000; Goldstein, 1985; Haggård-Grann, et al., 2006; Parker \& Auerhahn, 1998), la violencia al interior de las familias (Buckley et al., 2007 y 2013; Welsh \& Farrington, 2012) y la victimización (Foran \& O'Leary, 2008; Van Dijk \& De Waard, 1991) en una localidad del municipio de Guadalupe, Zacatecas, México. Dicha investigación consiste en un esfuerzo multidisciplinario de profesionales en políticas públicas, sociología, psicología y trabajo so-cial que buscan disminuir los factores que "expresan la existencia de conflictos y desequilibrios graves [y] que ad-vierten sobre el posible surgimiento de diversas formas de violencia" (Jusidman et al., 2011, p. 7).

Es decir, se trata de un proyecto que pretende articular la investigación académica y científica con la pre-vención social de la violencia. Una propuesta de esta envergadura implica la discusión especializada sobre los distintos diagnósticos de cada uno de los factores de riesgo (consumo de drogas, violencia contra las mujeres y victimización) en los que se pretende intervenir. Como primer paso en esa dirección, aquí se presenta un reporte breve de investigación referido a las prevalencias de consumo de drogas en población escolar de todas las escuelas secundarias y bachilleratos presentes en la localidad objeto de estudio.

Las prevalencias de consumo no son suficientes para hacer un diagnóstico sobre las adicciones; sin embargo, permiten una exploración inicial del fenómeno con miras a estudios posteriores. Dicho lo anterior, el presente reporte toma como base la discusión expuesta en Villatoro et al. en donde se plantea la necesidad de conocer la magnitud del problema del consumo de drogas en relación con "la prevalencia de por vida, en el último año, por grupos de edad y sexo [con el propósito de servir] como herramienta de análisis para ampliar las acciones preventivas basadas en evidencia" (Villatoro et al., 2016, p. 194). Este estudio es el punto de partida de la exposición.

\section{MÉTODO}

La técnica para la recolección de datos fue la aplicación de un censo. La población estadística estuvo constituida por los estudiantes de secundaria y bachillerato de la localidad seleccionada que se distribuyen en una escuela secundaria técnica, una preparatoria universitaria y una preparatoria estatal, todas públicas. El criterio para delimitar el conjunto de los sujetos de estudio fue que estuvieran inscritos en alguno de esos centros escolares, de modo que se recurrió a los registros administrativos de las instituciones para determinar a la población objetivo.

Se optó por el censo debido a que el total de sujetos era un número manejable $(N=1139)$ y el instrumento de recogida de información estaba diseñado para ser un autoreporte. Lo anterior permitió alcanzar una cobertura de 96.6\% (Tabla 1). El rechazo a responder el cuestionario fue mínimo. Una ventaja de los censos frente a las encuestas es que no se necesitan multiplicar los resultados por un factor de expansión, dado que éstos provienen de la población total, y además pueden considerarse altamente confiables porque se evitan errores aleatorios.

Matrícula y cuestionarios respondidos por escuela. Localidad en Guadalupe, Zacatecas, México, 2019

\begin{tabular}{llc} 
Tabla 1 Escuela & Matrícula & Cuestionarios respondidos \\
\hline Secundaria & 592 & 572 \\
Preparatoria estatal & 404 & 380 \\
Preparatoria universitaria & 143 & 148 \\
Total & 1139 & 1100 \\
\hline
\end{tabular}

Nota: Los datos de la matrícula fueron proporcionados por las propias autoridades educativas. Los autores de este reporte no tuvieron acceso a las listas oficiales de asistencia. 
Se aplicó el cuestionario de la Encuesta Nacional de Consumo de Drogas en Estudiantes (ENCODE), que diseñó el Instituto Nacional de Psiquiatría "Ramón de la Fuente Muñiz" para la Encuesta Nacional de Adicciones 2014 (Villatoro et al., 2016), en su versión para población escolar de secundaria y bachillerato. Cabe señalar que, si bien se aplicó el cuestionario completo sólo se exponen los resultados relativos a las prevalencias y niveles de riesgo, los cuales son preliminares de acuerdo con el esquema propuesto en Villatoro et al. (2016).

Como se señaló, dicho cuestionario es un instrumento de autoreporte; sin embargo, para su levantamiento, se solicitó el apoyo de un grupo de estudiantes universitarios de Ciencias Sociales de la Universidad Autónoma de Zacatecas, quienes recibieron un taller de capacitación sobre el propósito de la investigación, el objetivo, el procedimiento y la conclusión del levantamiento y la estructura, el contenido y los conceptos del instrumento.

\section{Consideraciones éticas}

El estudio fue aprobado y presentado ante autoridades locales, educativas y padres de familia. Se señaló que todas las respuestas al cuestionario serían estrictamente confidenciales y que ninguna persona podría ser identificada. Se insistió en que la participación era voluntaria y que ningún estudiante estaba obligado a responder el cuestionario. En la exposición de resultados se omite el nombre de la localidad y las escuelas participantes. Este estudio no forma parte del tipo de proyectos o protocolos de investigación referidos en la NOM-012SSA3-2012, toda vez que no se emplean medicamentos o materiales en seres humanos; asimismo, tampoco se refiere a la NOM-028-SSA2-1999, porque el levantamiento del censo no consiste en una medida de experimentación, prevención o control.

\section{RESULTADOS}

El $53.7 \%$ de la población estudiada eran mujeres y $46.3 \%$ hombres. El promedio de edad fue de 14.2 años. El 52\% de la población realizaba estudios de secundaria y $48 \%$ de bachillerato. En la secundaria, 19.4\% cursaba primer grado, $18 \%$ segundo y $14.6 \%$ tercero. En lo que corresponde a bachillerato, la distribución de los estudiantes en primero, segundo y tercer grado fue de 16.9, 16.1 y $14.9 \%$ respectivamente.

El $86.6 \%$ de la población eran estudiantes de tiempo completo, $9.3 \%$ estudiantes de medio tiempo y $4.1 \%$ afirmó no haber sido estudiante el año previo al censo. En cuanto a su ocupación laboral, $68.1 \%$ de los estudiantes no trabajaron, $19.8 \%$ trabajaron medio tiempo y $8.7 \%$ trabajó tiempo completo el año anterior al levanta- miento. Finalmente, con relación a su estabilidad emocional y física, $15.9 \%$ de los estudiantes declaró tener algún problema físico o emocional, como dificultades de aprendizaje, enfermedades físicas o mentales y problemas de conducta

\section{Prevalencias de consumo de alcohol}

La edad promedio de inicio de consumo de alcohol fue de 13.7 años, 13.4 en hombres y 14 en mujeres. El $49.4 \%$ de los estudiantes afirmó haber bebido "una copa completa de alguna bebida alcohólica" alguna vez en su vida; $42.6 \%$ hombres y $57.4 \%$ mujeres. Las prevalencias por escuelas fueron de $33 \%$ en secundaria, $62.8 \%$ en la preparatoria universitaria y $68.7 \%$ en la preparatoria estatal.

\section{Consumo en los últimos doce meses}

El 56.9\% de los estudiantes declaró haber consumido alcohol en los últimos 12 meses. Las prevalencias por escuela fueron de $13.1 \%$ en secundaria, $44.5 \%$ en la preparatoria universitaria y $48.1 \%$ en la preparatoria estatal.

\section{Consumo en el último mes}

El 35.8\% de los estudiantes afirmó haber consumido al menos una copa completa de alcohol en los últimos 30 días; $46 \%$ hombres y $54 \%$ mujeres. Las prevalencias por escuela fueron de $18.2 \%$ en secundaria, $25.5 \%$ en la preparatoria universitaria y $21.9 \%$ en la preparatoria estatal.

\section{Consumo excesivo de alcohol}

Tomando como referencia cuatro o más "copas de cualquier bebida alcohólica en una sola ocasión" en el último mes, el consumo excesivo se presentó en $27.3 \%$ de los estudiantes; $50.2 \%$ hombres y $49.8 \%$ mujeres. Por nivel educativo, este consumo se presentó en el 10.5\% de estudiantes de secundaria, $42.8 \%$ de la preparatoria universitaria y $46.2 \%$ de la preparatoria estatal.

\section{Niveles de riesgo}

El 27.8\% de los estudiantes presentó indicadores de inicio o presencia de dependencia al alcohol ${ }^{1}$, 52\% en hombres y $47.4 \%$ en mujeres. El $15 \%$ de estudiantes de secundaria presentó esta condición; lo mismo que $41.7 \%$ de estudiantes de preparatoria. En cuanto a las necesidades de atención por consumo de alcohol, el $47.2 \%$ de los estudiantes se ubicó en el nivel I de la prueba Alcohol Use Disorders Identification Test (AUDIT) de la Organización Mundial de la Salud, 38\% en el nivel II, $7 \%$ en el nivel III y $7.8 \%$ en el nivel IV. 


\section{Prevalencia de consumo de tabaco}

La edad inicial de consumo de tabaco fue 13.3 años, 13.1 años en hombres y 13.5 en mujeres. El promedio de cigarros consumidos al día fue 3.2. En la localidad, 8.3\% de los estudiantes fue identificado como fumador diario; 2 $63.7 \%$ hombres y $36.3 \%$ mujeres.

\section{Consumo alguna vez en la vida}

El $37.6 \%$ de los estudiantes declaró haber fumado alguna vez en la vida; $54.3 \%$ hombres y $45.7 \%$ mujeres. Las prevalencias por escuela fueron de $22.1 \%$ en secundaria, $50.7 \%$ en la preparatoria universitaria y $56.1 \%$ en la preparatoria estatal.

\section{Consumo en los últimos 12 meses}

El $20.1 \%$ de estudiantes respondió haber fumado en los últimos 12 meses; $57.6 \%$ hombres y $42.4 \%$ mujeres. Las prevalencias por escuela fueron de $9.1 \%$ en secundaria, $30.2 \%$ en la preparatoria universitaria y $32.8 \%$ en la preparatoria estatal.

\section{Consumo en el último mes}

El 9.1\% declaró haber consumido tabaco en el último mes; $71.9 \%$ hombres y $28.1 \%$ mujeres. Las prevalencias por nivel educativo fueron de $3 \%$ en secundaria, $16.1 \%$ en la preparatoria universitaria y $15.3 \%$ en la preparatoria estatal.

\section{Prevalencias de consumo de drogas}

El 33.3\% de los estudiantes afirmó que les habían ofrecido drogas regaladas y $7.9 \%$ afirmó que les han ofrecido drogas vendidas. En cuanto a edad de inicio del consumo, ésta fue de 12 a 15 años en las distintas drogas incluidas en el instrumento.

\section{Consumo alguna vez en la vida}

El $24.3 \%$ de los estudiantes consumió al menos una droga alguna vez en su vida, ya fuesen anfetaminas, tranquilizantes, mariguana, cocaína, crack o piedra, inhalables, metanfetaminas o heroína; $52.6 \%$ hombres y $46.6 \%$ mujeres. Las prevalencias por escuela fueron de $13.6 \%$ en secundaria, $37.4 \%$ en la preparatoria estatal y $31.1 \%$ en la preparatoria universitaria. La droga de mayor consumo fue la mariguana, $15.5 \%$; seguida de los tranquilizantes, $5.2 \%$, y los inhalables, $4.3 \%$ de prevalencia alguna vez en la vida.

\section{Consumo en los últimos 12 meses}

El $13.2 \%$ de los estudiantes consumió al menos una droga en los últimos 12 meses; $53.8 \%$ hombres y $45.5 \%$ mujeres. Las prevalencias por nivel escuela fueron de $6.8 \%$ en la secundaria, $17.6 \%$ en la preparatoria universitaria y $21 \%$ en la preparatoria estatal.

\section{Consumo en el último mes}

El 9\% de los estudiantes declaró haber consumido al menos una droga (exceptuando alcohol y tabaco) en el último mes; $62.6 \%$ hombres y $36.4 \%$ mujeres. Las prevalencias por escuela fueron de $4.7 \%$ en secundaria, $12.2 \%$ en la preparatoria universitaria y $14.2 \%$ en la preparatoria estatal.

\section{CONCLUSIONES Y DISCUSIÓN}

Los resultados preliminares del censo permiten una aproximación al problema del consumo de drogas en la localidad. En primer lugar, destaca que la tercera parte de los estudiantes tuvo acceso a drogas regaladas o vendidas. De acuerdo con trabajo de campo de corte cualitativo, realizado previamente, dicho acceso podría deberse a la presencia de organizaciones criminales en la localidad desde hace una década. Esto supondría un reto en términos de gobernabilidad, porque implica intervenir en un entorno social en condiciones de conflicto e ilegalidad. En segundo lugar, la encuesta arrojó que los estudiantes de la preparatoria estatal tienen prevalencias de consumo notablemente más elevadas que los estudiantes de la preparatoria universitaria. Esto podría deberse a que estos últimos suelen provenir de rancherías, en donde se presume que el acceso a drogas es menor. En tercer

\footnotetext{
${ }^{1}$ Con base en la prueba AUDIT de la OMS, entendemos que la presencia o el inicio de la dependencia al alcohol se indica cuando se responde "menos de una vez al mes", "mensualmente", "semanalmente" o "diario o casi diario" en cualquier de las siguientes preguntas: Durante los últimos 12 meses, ¿con qué frecuencia?..

a) ¿No pudiste parar de beber una vez que habías empezado?

b) ¿Dejaste de hacer algo que deberías de haber hecho por beber?

c) ¿Bebiste en la mañana siguiente después de haber bebido en exceso el día anterior?

2 Entendemos por fumadores diarios aquellos que respondieron al menos "1" a la pregunta “¿Cuántos cigarros fumas al día?”.
} 
lugar, se subraya que no se perciben mayores diferencias de consumo entre hombres y mujeres, lo que resulta coherente con resultados nacionales (Villatoro, et al., 2016); a este respecto, cabe recalcar la importancia de diseñar medidas con enfoque de género y apoyo en los derechos humanos.

De acuerdo con estos resultados, se advierte la necesidad de identificar factores asociados al consumo de drogas, como los problemas emocionales y otros temas incluidos en el resto de las secciones del cuestionario que no han sido incorporadas en este artículo, y que deberán ser discutidas en otro momento. Un análisis exploratorio de regresión logística bivariado podría ser pertinente para estudiar la relación entre las características personales o sociodemográficas y el consumo de drogas.

En cuanto a las medidas de intervención, se propone explorar dos cursos de acción complementarios, uno de tipo psicosocial y otro de corte sociopolítico. En cuanto a la intervención psicosocial, se podrían diseñar intervenciones breves, adaptadas a la población estudiada, que sean probadas en grupos pilotos. Debido a que se trata de una población joven, es posible discutir un enfoque de psicología positiva (Seligman et al., 2005) que promueva comportamientos tendientes a generar balance emocional y cultivar las fortalezas de carácter (Park et al., 2004). ${ }^{3}$ En cuanto a la ruta sociopolítica, debe advertirse la necesidad de articular un trabajo de intervención a mediano y largo plazo con la colaboración del gobierno, las autoridades educativas y los padres de familia para contener los efectos del entorno social sobre los estudiantes. La implementación de ambas rutas, aunadas a acciones para prevenir el resto de los factores de riesgo de la violencia identificados previamente, podrían contribuir a mejorar las condiciones de vida generales de la localidad.

\section{FINANCIAMIENTO}

Este reporte fue elaborado con recursos del Programa de Apoyo a Proyectos de Investigación e Innovación Tecnológica de la Universidad Nacional Autónoma de México, PAPIIT-UNAM IA300118.

\section{CONFLICTO DE INTERÉS}

Los autores declaran que no tienen conflicto de intereses.

\section{AGRADECIMIENTOS}

Las autoridades ejecutivas, legislativas y educativas en la localidad objeto de estudio brindaron las facilidades operativas para la realización del estudio. Agradecemos a Yéssica Sánchez su apoyo en el procesamiento de la información.

\section{REFERENCIAS}

Buckley, H., Holt, S., \& Whelan, S. (2007). Listen to me! children's experiences of domestic violence. Child Abuse Review, 16(5), 296-310. doi: 10.1002/car.995

Buckley, H., Holt, S., \& Whelan, S. (2013). Children's experiences of domestic violence en K. Safford, M. Stacey \& R. Hancock (Eds.), Small-Scale Research in Primary Schools: A Reader for Learning and Professional Development. Routledge. doi: $10.4324 / 9781315881225$

Carrión, F. (2005). La inseguridad ciudadana en América Latina. Quórum. Revista de Pensamiento Iberoamericano, 12, 29-52.

Dammert, L. (2007). Perspectivas y dilemas de la seguridad ciudadana en América Latina. FLACSO Ecuador. https://biblio.flacsoandes.edu.ec/catalog/resGet.php?resld=40089

Duke, A., Smith, K., Oberleitner, L., Westphal, A., \& McKee, S. (2017). Alcohol, Drugs, and Violence: A Meta-Meta-Analysis. Psychology of Violence, 8(2), 238-249. doi: 10.1037/vio0000106

Fergusson, D. M. \& Horwood, L. J. (2000), Does cannabis use encourage other forms of illicit drug use? Addiction, 95(4), 505520. doi: 10.1046/j.1360-0443.2000.9545053.x

Foran, H. M., \& O'Leary, K. D. (2008). Alcohol and intimate partner violence: A meta-analytic review. Clinical Psychology Review, 28(7), 1222-1234. doi: 10.1016/j.cpr.2008.05.001

Gander, F., Proyer, R. T., \& Ruch, W. (2016). Positive Psychology Interventions Addressing Pleasure, Engagement, Meaning, Positive Relationships, and Accomplishment Increase Well-Being and Ameliorate Depressive Symptoms: A Randomized, Placebo-Controlled Online Study. Frontiers in Psychology, 7, 686. doi; 10.3389/fpsyg.2016.00686

Gander, F., Proyer, R. T., Ruch, W., \& Wyss, T. (2013). Strength-based positive interventions: further evidence for their potential in enhancing well-being and alleviating depression. Journal of Happiness Studies, 14(4), 1241-1259. doi: 10.1007/s10902-012-9380-0

Goldstein, P. J. (1985). The drugs/violence nexus: A tripartite conceptual framework. Journal of Drug Issues, 15(4), 493-506. doi: 10.1177/002204268501500406

Haggård-Grann, U., Hallqvist, J., Långström, N., \& Möller, J. (2006). The role of alcohol and drugs in triggering criminal violence: a case-cros-

\footnotetext{
${ }^{3}$ Las intervenciones que modelan y moldean las fortalezas de carácter han tenido un éxito particular al mostrar evidencia de que a través de entrenamientos breves simultáneamente se incrementa el bienestar subjetivo y disminuyen los trastornos de ansiedad y depresión, y que esos cambios pueden permanecer por varios meses sin que se administre mantenimiento explícito (Gander, Proyer \& Ruch, 2016; Gander, Proyer, Ruch, \& Wyss, 2013; Proyer, Gander, Wellenzohn \& Ruch, 2015).
} 
sover study. Addiction, 101(1), 100-108. doi: 10.1111/j.13600443.2005.01293.x

Jusidman, C., Rubalcava, R.M., Carreón I.G, Marín, O.E. \& Stern, A. (2011). Nota metodológica para el diagnóstico territorial de las causas sociales de las violencias. Iniciativa Ciudadana y Desarrollo Social, INCIDE Social, A.C.

Murphy, A., Taylor, E., \& Elliott, R. (2012). The detrimental effects of emotional process dysregulation on decision-making in substance dependence. Frontiers in Integrative Neuroscience, 6, 101 doi: 10.3389/fnint.2012.00101

Park, N., Peterson, C., \& Seligman, M. E. P. (2004). Strengths of character and well-being. Journal of Social and Clinical Psychology, 23(5), 603-619. doi: 10.1521/jscp.23.5.603.50748

Parker, R. N., \& Auerhahn, K. (1998). Alcohol, Drugs, and Violence. Annual Review of Sociology, 24, 291-311. doi: 10.1146/annurev. soc.24.1.291

Proyer, R. T., Gander, F., Wellenzohn, S., \& Ruch., W. (2015). Strenghts-based positive psychology interventions: a randomized placebo-controlled online trial on long-term effects for a signature strengths- vs. a lesser strengths-intervention. Frontiers in Psychology, 6, 456. doi: 10.3389/fpsyg.2015.00456
Rico, J. M., \& Chinchilla, L. (2002). Seguridad ciudadana en América Latina: hacia una política integral. Siglo XXI.

Seligman, M. E. P., Steen, T. A., Park, N., and Peterson, C. (2005). Positive psychology progress: empirical validation of interventions. The American Psychologist, 60(5), 410-421. doi.org/10.1037/0003-066X.60.5.410

Oficina de Naciones Unidas contra la Droga y el Delito (2011). Manual sobre la aplicación eficaz de las directrices para la prevención del delito. Naciones Unidas. https://www.unodc.org/documents/ justice-and-prison-reform/crimeprevention/Handbook_on_the_ Crime_Prevention_Guidelines_Spanish.pdf

Van Dijk, J. J. M., \& De Waard, J. (1991). A two-dimensional typology of crime prevention projects. Criminal Justice Abstracts, 483-503.

Villatoro Velázquez, J. A., Medina-Mora Icaza, M. E., Martín del Campo Sánchez, R., Fregoso Ito, D. A., Bustos Gamiño, M. N., Resendiz Escobar, E., Mujica Salazar, R., Bretón Cirett, M., Soto Hernández, I. S., \& Cañas Martínez, V. (2016). Drugs use in Mexican students: trends and Magnitude of the problem. Salud Mental, 39(4), 193-203. doi: 10.17711/SM.0185-3325.2016.023 Welsh, B. C., \& Farrington, D. P. (2012). The Oxford Handbook of Crime Prevention. Oxford University Press. doi: 10.1093/oxfordhb/9780195398823.013.0001 